\title{
LA PENÍNSULA DORADA. FUENTES LITERARIAS PARA EL ESTUDIO DE LOS RECURSOS AURÍFEROS DE IBERIA/HISPANIA
}

\author{
Jordi Pérez González ${ }^{1}$ \\ https://doi.org/10.18778/8220-421-6.08
}

\begin{abstract}
The first contacts of the Greeks and Phoenicians with the Iberian Peninsula revealed the importance of the resources of the Iberian populations, especially in terms of metals. For decades, this news persuaded the more adventurous to undertake a trip to the West in search of fortune. On many occasions these stories were exaggerated, overvaluing the wealth existing with in the limits of the western Ecumene, and encouraging the creation of a literary topos around the descriptions of the territory.

The Roman expansion through the western Mediterranean led to a series of references to the peninsular's wealth by several authors in the service of Rome. They detailed in general the geography, populations and wealth that one could find in these regions.
\end{abstract}

1 Universitat de Girona. Miembro del grupo CEIPAC y del Instituto de investigación UBICS. jordi.perezgonzalez@udg.edu. Proyecto financiado por: Ministerio de Ciencia e Innovación: Juan de la Cierva-Formación (2019. REF: FJC2019-040688-I); Relaciones Interprovinciales en el Imperio Romano. Producción y comercio de alimentos hispanos (Provincia Baetica et Tarraconensis) (HAR201785635-P); Grup de Recerca en Arqueologia, Prehistòria i Patrimoni (GREPPAA) (2017SGR-1688) y Dinàmiques socioeconòmiques del món rural romà: formes d'hàbitat i cultura material al litoral central català (CLT009/18/00045). 
In the present work, we have gathered together those literary sources linked to the descriptions of gold in the landscapes of the Iberian Peninsula. As I have commented in some of my works, the acquisition of gold by Rome occupied much of its history on the Peninsula. Gold, as a positional good, is a scarce good by nature, which cannot be created, only redistributed. Pliny commented that the amount of gold known in the ancient world was always less than that of other precious metals (Plin. Nat. 33.16). By pointing out the news referred to in the Roman world about obtaining Hispanic resources, we will indirectly address what was the perception that Rome had about Iberia / Hispania.

Keywords: Iberia, Hispania, mines, gold, literary sources.

Palabras clave: Iberia, Hispania, minas, oro, fuentes literarias.

Muy pronto, los primeros contactos de los pueblos griegos con la Península Ibérica revelaron las riquezas del territorio y las grandes oportunidades comerciales que ofrecía el territorio. Por Heródoto sabemos de la buena fortuna de los primeros griegos al hallar en el extremo Occidente a los tartesos, de cuyo intercambio comercial obtuvieron pingües beneficios:

Y como el aire no amainó, atravesaron las Columnas de Hércules y, bajo el amparo divino, llegaron a Tarteso. Por aquel entonces, aquel emporio comercial estaba sin explotar, de manera que, a su regreso a la patria, los samios, con el producto de su flete, obtuvieron más beneficios que cualquier otro griego. (Hdt. 4, 142)

Los habitantes de Focea, por cierto, fueron los primeros griegos que realizaron largos viajes por el mar y son ellos quienes descubrieron el Adriático, Tirreno, Iberia y Tartesso. No navegaban en naves mercantes, sino en pentaconteros [barco de guerra griego de 50 hombres, de ahí su nombre]. Y, al llegar a Tartesso, se hicieron muy amigos del rey de los tartesios, cuyo nombre era Argantonio, que gobernó Tartesso durante ochenta años y vivió en total ciento veinte. (Hdt. 1, 163) 
Sin adentrarnos en la supuesta relación de la figura del rey Argantonio con la plata y su consecuente ciclo mítico, son varias las fuentes griegas que relacionan Tarteso directamente con su riqueza argentífera (Hecat. 45 Nenci apud St. Byz; Ps.-Arist. Mir. 135; Strab. 3, 2, 11; St. Byz. 1, 606 Meineke), o por el hallazgo de estaño, oro y/o cobre en su territorio (Ps.-Scymn., Orbis descriptio 164-166) (todo ello en: Padilla Monge, 2014: 12ss). En esta línea, son varias las referencias sobre la riqueza metalífera del suelo hispano, siendo de gran detalle las menciones de autores griegos y romanos sobre las explotaciones auríferas.

De sur a norte, ya Turdetania, región coincidente con la civilización de pueblos tartésicos, fue descrita por Estrabón como el lugar conocido con mayor abundancia en lo que a oro, plata, cobre y hierro se refiere, tanto en su cantidad, como en su calidad (Strab. 3.2.8). Más tarde, Plinio también coincidió al destacar la calidad y delicadeza tanto de la plata hispana, como de su oro (Plin. Nat. 33.34). En las comarcas Ilipa y Sisapon se halló plata y cerca de Cotinas (Kotinai) se halló oro junto al cobre (Strab. 3.2.8). En ocasiones estas minas de cobre fueron conocidas como minas de oro, de lo que se infiere que anteriormente se extraía de ellas oro (Strab. 3.2.8). Una noticia similar la recoge Pausanias al comentar que los iberos encuentran el cinabrio juntamente con el oro (Paus. 8.39.5-6). No hay que olvidar que la composición del cinabrio, bermellón o minium, como fue conocido en época romana, era de un $85 \%$ de mercurio, por un $15 \%$ de azufre. Siendo el empleo de este mineral necesario para los procesos de depuración del oro mediante la técnica de la amalgama (Plin. Nat. 33, 32, Vitr. De Architectura. 7. 8, 4 y Strab. 3.2.8; recogido en Chic García, 1991: 12-17; cfr. Rodríguez Almeida, 1986: 49-60; 1994: 335-345)².

2 Teofrasto declara que, noventa años antes de la magistratura de Praxibulus en Atenas - una fecha que responde al año de nuestra ciudad, 439- el minio (vel cinabrio) fue descubierto por Calias el ateniense, quien tenía la esperanza de extraer oro al someter la arena roja que encontró en las minas de plata a la acción del fuego. Plin. Nat. 33.41. También es Plinio quien refiere el minio como una 'invención griega', probablemente introducido en Occidente por los griegos; Plin. Nat. 33.37. En: Rodríguez Almeida, 1994: 335ss, notas 1-3. 
Y solo después de la segunda guerra púnica y del descubrimiento y explotación del miniarum Sisaponense de la Baetica ${ }^{3}$ el minio efesio dejó de tener exclusividad en Roma, donde sería trasladado para su empleo en las officinae minii de la capital. En estas, Rodríguez Almeida creyó en la posibilidad de que Roma llegó a convertirse en el "mayor centro de producción (y por lo tanto de comercialización) del mercurio" (Rodríguez Almeida, 1994: 335-345). Es de notar que, en base a mis últimas investigaciones sobre el comercio de oro en Roma, comparándolo también con el resto de territorios, me atrevería a adelantar que seguramente también tuvo la primacía de la elaboración y comercialización del oro (Pérez González, 2017: 37-70; en prensa).

Pronto, la fama del subsuelo turdetano (e hispano) superó situaciones reales y en ocasiones fue presentada por los autores clásicos a través de episodios fantásticos, convirtiéndose en un topos literario que animó la imaginación de conquistadores y buscavidas. Por ejemplo, cuenta Estrabón que Posidonio, para ensalzar el número de minas y la riqueza del territorio turdetano se entusiasmó mediante el uso de hipérboles al narrar un episodio, cuando "una vez se incendiaron los bosques, la tierra que era de plata y oro, se fundió hirviendo a la superficie, porque cada monte y cada colina eran materia de moneda acumulada por un azar generoso..." (Strab. 3.2.9). Otra noticia, esta vez de Apiano, recuerda el episodio vivido por el general romano Lucio Licinio Lúculo cuando al hacer inventario del botín obtenido de la toma de Intercatia (151 a.C.) quedó defraudado al ver que no había entre él ni el oro, ni la plata que hacían famosa a Iberia, razón por la cual Lúculo "precisamente hacía la guerra" (App. Hisp. 9.54). Al parecer, el hecho de que los vacceos no poseyeran estos metales fue entendido por Apiano como la falta de interés de estos celtiberos por la posesión de estos productos. Por Estrabón conocemos la preferencia por el trueque de mercancías de las poblaciones montañesas interiores ${ }^{4}$ al empleo de metales preciosos (Strab. 3. 3. 7).

3 También Plinio hace referencia a la riqueza en minio de la Baetica. Plin. Nat. 3.3 (4). 30-33.

4 Galaicos, astures, cántabros, vascones y pirenaicos. 
Aun así, cuando se decidieron por el uso de metales preciosos en el comercio, por norma utilizaron recortes de láminas de plata, hecho común entre muchas poblaciones de la antigüedad, quienes valoraban los metales preciosos su valor intrínseco, es decir, basándose en el valor en el mercado del metal sin tener en cuenta si lo recibían acuñado en forma de moneda o manufacturado en algún objeto de prestigio. Una noticia similar fue recogida por Tácito al describir a los pueblos germanos con menos contacto con Roma, de quiénes dice, que no les afectaba la posesión de la plata y del oro, en este caso, porque la desconocían (Tac. Ger. 5.1-5).

En paralelo, muchas de las descripciones sobre la riqueza metalífera de la Península, además de comentar la existencia de montes ricos en metales, hablan de la existencia de arenas auríferas en los cursos fluviales más importantes, destacando la región rivereña que iría desde el río Guadiana hasta el Tajo (Strab. 3.2.8). Así, son varios los autores que destacan el carácter aurífero del río Tajo y de sus afluentes (Strab. 3.3.4; Catul. Carmina. 29; Luc. 7.728; Plin. Nat.33.22-25). Por su parte, Blázquez se hace eco de las fuentes que mencionan la existencia de ríos de la vertiente atlántica también conocidos por su riqueza aurífera (Strab. 3, 153; 4.208; 15, 718; Ius. 44.1.7; recogido en Blázquez Martínez 1982. De la versión digital, Gabinete de Antigüedades de la Real Academia de la Historia: 10).

Nuevamente Estrabón, pone el foco de atención, en esta ocasión en el sudeste peninsular, al comentar la existencia de minas de oro y otros minerales en la zona montañosa de Bastetania (Strab. 3.4.2).

Con la conquista romana de estos territorios y el acceso a sus recursos, Hispania se tornaba, gracias a sus minas, en reserva estratégica para el numerario romano. Si ponemos de ejemplo la exportación de las minas de Carthago Nova, ya bajo dominio cartaginés, las minas argentíferas de la zona reportaban 50 toneladas anuales de metales, cifras que debieron ser similares durante la dominación romana, cuando llegaron a movilizarse hasta 40.000 esclavos. Así, la continuada sobreexplotación de las minas del territorio originó un temprano agotamiento de las mismas (Ferrer Maestro, 2005: 145). 
Pocos años después de la conquista de estos territorios, la explotación sistematizada de las minas hispanas resultó una oportunidad para el enriquecimiento de muchos romanos, destacando entre todos ellos a Sexto Mario, quien, según Tácito, fuese el hombre más rico de Hispania (Tac. Ann. 6.19).

Y fue en la zona más septentrional de la Península en el territorio que va desde los Pirineos hasta Asturias, Gallaecia y Lusitania donde Plinio mencionó la existencia de las minas más ricas de Hispania, donde destacó el hallazgo de oro, así como de plata, hierro, plomo y estaño (Plin. Nat. 4.20 [34]. 112; 33.22-25; 33.27).

A continuación, cabe recordar las noticias ofrecidas por otros autores que coinciden con el testimonio sobre la abundancia metalífera de Iberia/Hispania: Apiano (App. Hisp. 9.54), Ateneo (Ath. 2.21), Estrabón (Strab. 11.2.19), Lucano (Luc. 7.728) ${ }^{5}$, Mela (2.86), Plinio (Plin. Nat. 3.3 (4). 30-33; 33.22-25) y Polibio (Plb. 3.57.1-4).

En esta línea, es Estrabón quien comenta la posibilidad de que el pueblo de los iberos recibiera su nombre por la existencia de minas de oro, como los iberos del este (Strab. 11.2.19). Plinio habla de los valos y suanos, pueblos que se dedican a la extracción de oro más allá de las Puertas Caucásicas, en lo que fue la Iberia del este o del Ponto (Plin. Nat. 6.11 [12]). Este hecho bien pudo responder a la realidad de ambos territorios, o quizás solo se trate de la duplicidad toponímica propia de la expansión griega en los límites de la ecúmene. Una visión acertada sobre ello es comentada por Padilla Monge al concretar que:

uno de los fundamentos de la geografía arcaica griega era la creencia en la existencia de simetrías y de correspondencias entre los extremos del mundo, pues se pensaba que la tierra habitada estaba dividida en porciones simétricas, tanto en sentido

5 Luc. 7.728. Según la traducción y las notas de A. Holgado Redondo para la edición Gredos, cuando se menciona aquí el oro arrebatado a los pueblos de Hesperia, se trata de Hispania, aunque generalmente designe Italia. $C f$. nota 646 de Lucano. Farsalia. Traducción y notas de A. Holgado Redondo. Ed. Gredos. 1982. Edición 2008. 
norte-sur como en sentido este-oeste (Moret, 2006: 54). Este último eje, en el que se imponía la idea esquemática bipolar de que el estrecho de Gibraltar y el Bósforo, que marcaban unos importantes hitos del mundo conocido, debían ser iguales y simétricos (Gangutia, THA 1998: 255), resultó bastante productivo desde el punto de vista de los dobletes toponímicos. (Padilla Monge, 2014: 11-12)

En relación con el tema y el marco geográfico del concepto 'Iberia' e 'Ibero' destacan los trabajos de Domínguez Monedero (1983: 203-224), Gómez Fraile (1999: 159-187) y Celestino, LópezRuiz (2016: 58, nota 24); quienes a su vez aluden a los trabajos de Cunchillos y de Hoz para proponer un origen fenicio-púnico del nombre de Hispania, destacando tan solo su valor geográfico ('isla', 'costa' del 'norte') y obviando otra de las interpretaciones de Cunchillos y Zamora donde proponen para Hispania el desarrollo I+span+ya, 'costa de metales', o 'costa de las forjas de metales', o 'costa de los forjadores', o incluso 'isla donde se chapean o baten metales' (Cunchillos, Zamora, 1997: 141-154). Como apuntase Blázquez, esta explicación parece más aceptable, ya que los fenicios venían a Hispania por metales, que intercambiaban en el Mediterráneo oriental, como comenta Diodoro Sículo (5.35.3, cf. Blázquez Martínez, 2005: 3). En este punto parece fácil pensar que tanto griegos como fenicios al comprobar la riqueza en metales de la Península coincidiesen al recordar a estos nuevos territorios por sus bienes. Por el momento, no tengo noticia que se haya propuesto nada parecido, aunque mi desconocimiento no quiere decir que no se haya hecho.

En gran medida, muchas de estas noticias sobre las explotaciones mineras de la península ya fueron recogidas por Blázquez, quien en sus trabajos puso de manifiesto la riqueza metalúrgica del territorio y los muchos beneficios que le supuso su conquista a Roma. Para Blázquez:

[...] la mayor partida de ingresos que Roma recibía de la Península Ibérica procede de las explotaciones mineras. El Mediterráneo era muy pobre en minas, África no las tenía. Las minas 
de plata de Laurion en las proximidades de Atenas estaban en decadencia absoluta. Grecia sólo contaba con minas en las regiones de Tracia y Macedonia. Alguna plata tenía Cerdeña. Hispania fue para Roma, en frase del historiador Piganiol, el mayor distrito minero del imperio en formación y el primero que fue explotado. Hispania era El Dorado de Occidente, en opinión de Charles Picard (Blázquez Martínez, 1982) .

A este respecto, tanto el número de textos literarios, como el hallazgo arqueológico de una gran cantidad de estas minas (Fig. 1) confirmarían la propuesta de Blázquez, a la que nos sumamos.

La relación del oro como bien posicional es clave para entender la conquista de Hispania, su saqueo y la posterior organización de los territorios metalíferos con el objetivo de impulsar y controlar estos recursos estratégicos (Hirsch. 1976; Pérez González, 2017: 60; 2020). A día de hoy, son diversos los estudios dedicados a analizar el impacto de la conquista romana de Hispania, y las consecuencias de la política de explotación metalífera de la Península. Destacan los siguientes estudios: en primer lugar, los trabajos desarrollados dentro del proyecto AVREVS, cuyo objetivo es estudiar la caracterización y procedencia del oro de Occidente (Blet-Lemarquand, Suspène, Amandry, 2015: 112; Bocciarelli, Blet-Lemarquand, Suspène, 2015: 175-188), en segundo lugar, debemos señalar aquellos estudios vinculados con el control administrativo-militar del noroeste peninsular en época imperial, relacionado con el mantenimiento de las exploraciones mineras así como de su red de comunicaciones/intercambios

6 Blázquez Martínez 1982. De la versión digital, Gabinete de Antigüedades de la Real Academia de la Historia: 10. También Rostovtzeff 1937, 413 afirmó que "España era, en efecto, el distrito minero más rico del Imperio en formación y el primero que fue explotado. Por su parte, Blázquez ha reiterado la idea de que Hispania fue El Dorado para cartagineses y romanos en: Blázquez Martínez 1978a; 1978b. Sobre las minas en Hispania destacan los trabajos de Domergue 1990; 2008. Otras obras generales sobre el oro hispano y las minas de época romana: Davies 1935; Quiring 1948; Hirt 2010. 
(Morillo, 2014: 133-148; 101-132; De Soto, 2019; Costa-García, 2018: 986-993). En tercer lugar y en línea con los trabajos sobre la sistematización administrativa y urbana del Noroeste de Hispania, cf. Sastre, Orejas, Currás, Zubiaurre, 2017: 537-552, debemos estar atentos a los resultados del proyecto PATRIMONIVM (ERC-StG 716375) coordinado por el Dr. Alberto Dalla Rosa, donde se estudia la política, sociedad y rol económico de las propiedades imperiales. En alguno de sus trabajos, aún sin publicar, destacan mediante el análisis epigráfico una gran concentración de estas propiedades en el noroeste peninsular, hecho que podría vincularse con la explotación de las minas de oro de la región, un producto bajo el control directo del emperador (Pérez González, 2017: 37-70). Por último, resulta interesante mencionar los análisis realizados sobre la polución del plomo conservado en el hielo ártico, como consecuencia de su empleo en la industria minera, notándose un incremento de los procesos mineros a partir de la explotación de la Península por parte de cartagineses y romanos (Rosman, Chisholm, Hong, Candelone, Boutron, 1997: 3413-3416; McConnell, Wilson, Stohl, Arienzo, Chellman, Eckhardt, Thompson, Pollard, Steffensen, 2018: 5726-5731; Terpstra, 2019).

Para finalizar y hacernos una idea de la fama que obtuvo el oro hispano en el día a día de los romanos, sabemos por uno de los epigramas de Marcial, donde menciona el fragor de los talleres de batidores de metales ${ }^{7}$, del constante golpeo a la balux Hispana (Marcial. Epig. 12.57.9-10) $)^{8}$, entendiéndose como el oro que viene de Hispania, o mineral de oro hispano, como especificó Rodríguez Almeida, 1994: 339. Así, al hacer referencia del oro trabajado en la capital, Marcial especifica su procedencia como un reflejo de la cotidaneidad del asunto, a o bien, para mostrar una cualidad del metal descrito.

7 Sobre los batidores de metales o batihojas, s.v. brattiari en Pérez González, 2019.

8 Marcial. Epig. 12.57.7-10. "Hinc otiosus sordidam quatit mensam Neroniana nummlarius massa, illinc balucis malleator Hispanae tritum nitenti fuste uerberat saxum". 


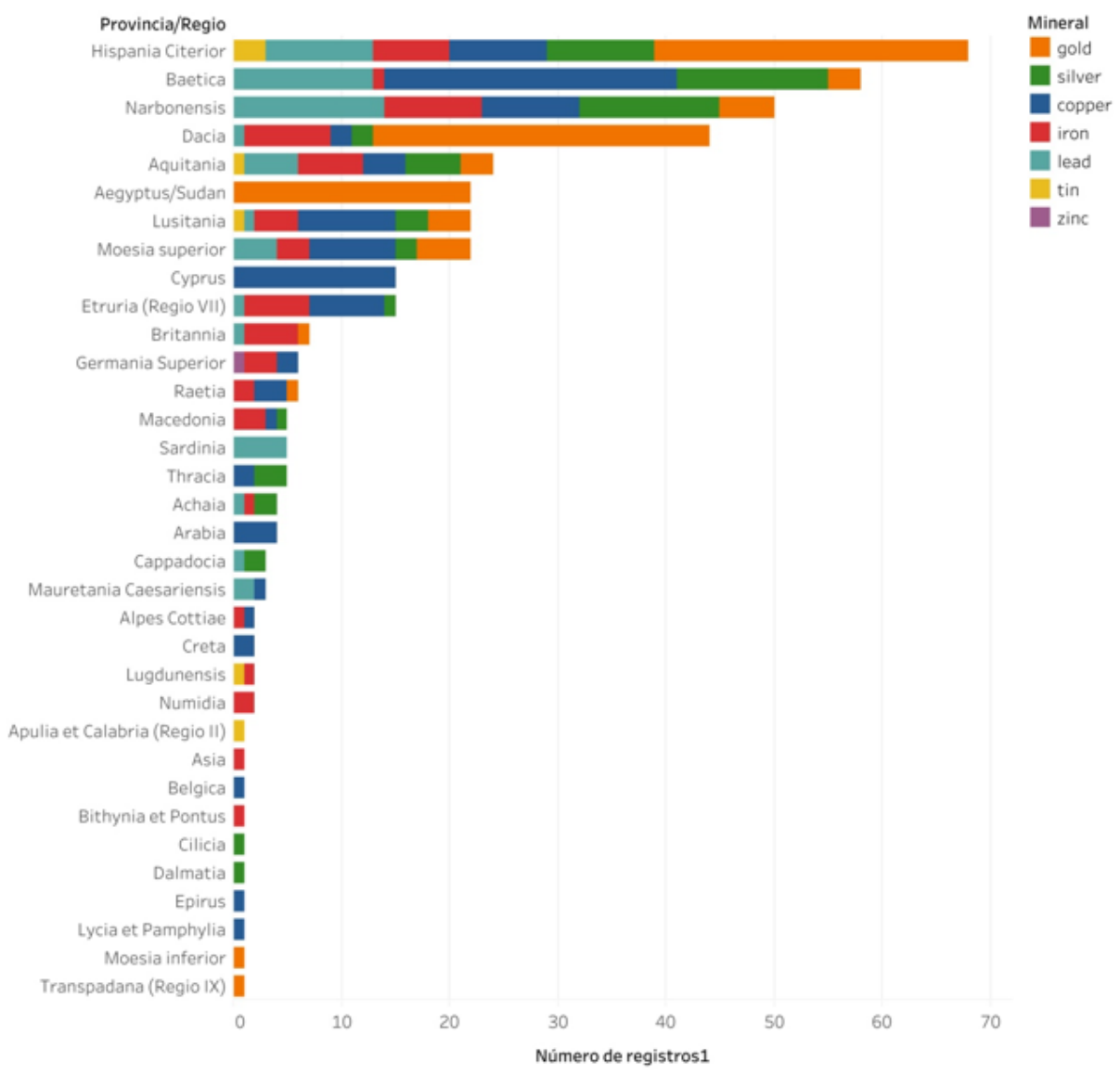

Figura 1. Minas romanas ${ }^{9}$.

9 Fuente de datos: Pleiades (https://pleiades.stoa.org). La edición del gráfico de barras de la situación de las minas por regiones itálicas y provincias romanas ha sido elaborada con el software de visualizaciones de Tableau (https://www.tableau.com). En el siguiente enlace puede visitarse el mapa de las minas de oro: https://jperezg1985.carto.com/builder/8171b72d-243f-4ff0-924d-bff482ebecbe/embed. Los datos empleados para las visualizaciones pueden descargarse en el repositorio de acceso libre Github; en el siguiente enlace: https:/github.com/JordiPerezGonzalez/Gold-mines.git 


\section{Bibliografía}

Blázquez Martínez, J. (1978a): Economía de la Hispania Romana. Bilbao: Nájera.

Blázquez Martínez, J. (1978b): Historia económica de la Hispania Romana. Madrid: Cristiandad.

Blázquez Martínez, J. (1982): "El sistema impositivo en la Hispania romana", en: Historia de la Hacienda Española (épocas antigua y medieval). Homenaje al Profesor García de Valdeavellano. Madrid: Ministerio de Hacienda, Instituto de Estudios Fiscales: 67-125.

Blázquez Martínez, J. (2005): "El nombre de Hispania en la Historia. Los Hispanos en el Imperio Romano", en: V. Palacio Atard (coord.) De Hispania a España: el nombre y el concepto a través de los siglos. Madrid: Temas de hoy: 17-39.

Blet-Lemarquand, M., Suspène, A., Amandry, M. (2015): "Augustus' gold coinage: investigating mints and provenance through trace elements concentrations", en: A. Hauptmann, D. Modarressi-Tehrani. (eds.), Archaeometallurgy in Europe III, Proceedings of the third International Conference, Deutsches BergbauMuseum Bochum, June 29 - July 1 2011. (Der Anschnitt, Beiheft 26). Bochum: Deutsches Bergbau-Museum: 107-113.

Bocciarelli, D., Blet-Lemarquand, M., Suspène, A. (2017): "Les monnaies d'or des années 68-69 p.C. frappées dans les provinces occidentales: l'apport de l'étude pondérale et des analyses élémentaires", en: L. Bricault, A. Burnett, V. Drost, A. Suspène (sous la direction de) Rome et les Provinces. Monnayage et Histoire. Mélanges offerts à Michel Amandry. Bordeaux: Ausonius Éditions: $175-188$.

Celestino, S., López-Ruiz, C. (2016): Tartessos and the Phoenicians in Iberia. Oxford: Oxford University Press.

Chic García, G. (1991): “Estrabón y la práctica de la amalgama en el marco de la minería sudhispánica: un texto mal interpretado", en: C. González Román (ed.) La Bética en su problemática histórica. Granada: Universidad de Granada: 7-30.

Costa-García, J.M. (2018): "Roman Camp and Fort Design in Hispania: An Approach to the Distribution, Morphology and Settlement Pattern of Roman Military Sites during the Early 
Empire”. En: C.S. Sommer, S. Matešić (eds.), Limes XXIII. Proceedings of the 23rd International Limes Congress in Ingolstadt 2015. Mainz: Nünnerich-Asmus. Vol. 2: 986-993.

Cunchillos, J.L., Zamora, J.A. (1997): Gramática elemental fenicia. Madrid: Consejo Superior de Investigaciones Científicas.

Davies, O. (1935): Roman Mines in Europe. Oxford: Arno Press.

De Soto, P. (2019): "Network Analysis to Model and Analyse Roman Transport and Mobility", en: P. Verhagen, J. Joyce, M. Groenhuijzen (eds.), Finding the Limits of the Limes. Computational Social Sciences. Springer: 271-289.

Domergue, Cl. (1990): Les mines de la Péninsule Ibérique dans l'Antiquité Romaine. Rome: Publications de l'École Française de Rome.

Domergue, Cl. (2008): Les mines antiques. La production des métaux aux époques grecque et romaine. Paris: Picard.

Domínguez Monedero, A.J. (1983): “Los términos 'Iberia' e 'Iberos' en las fuentes grecolatinas: estudio acerca de su origen y ámbito de aplicación". Lucentum 2: 203-222.

Ferrer Maestro, J.J. (2005): La República participada. Intereses privados y negocios públicos en Roma. (Col-lecció Humanitats, núm.16). Castellón de la Plana: Publicacions de la Universitat Jaume I.

Gómez Fraile, J.M. (1999): “Los conceptos de 'Iberia' e 'Ibero' en Estrabón”. SPAL 8: 159-187.

Hirsch, F. (1976): The social limits to growth. Londres: Routledge. Hirt, A.M. (2010): Imperial Mines and Quarries in the Roman World. Organizational Aspects 27 BC-AD 235. Oxford: Oxford University Press.

McConnell, J.R., Wilson, A.I., Stohl, A., Arienzo, M.M., Chellman, N.J., Eckhardt, S., Thompson, E.M., Pollard, A.M., Steffensen, J.P. (2018): "Lead pollution recorded in Greenland ice indicates European emissions tracked plagues, wars, and imperial expansion during antiquity". Proceedings of the National Academy of Sciences May 2018, 115 (22): 5726-5731.

Moret, P. (2006): “La formation d'une toponymie et d'une ethnonymie grecques de l'Ibérie: étapes et acteurs”, en: G. Cruz 
Andreotti, P. le Roux, P. Moret (eds.), La invención de una geografía de la Península Ibérica I. La época republicana. Málaga-Madrid: Diputación de Málaga, Centro de Ediciones de la Diputación de Málaga (CEDMA): Casa de Velázquez: 39-76.

Morillo, A. (2014): “Arqueología de la conquista del norte peninsular. Nuevas interpretaciones sobre las campañas del 26-25 a.C». En: F. Cadiou, M. Navarro (eds.) La guerre et ses traces. Conflicts et Sociétés en Hispanie à l'époque de la conquête romaine (IIIe-Ier siècle av. J.C.) Bordeaux: Ausonius éditions. Mémoires 37: 133-148.

Padilla Monge, A. (2014): "Algunas notas sobre la figura de Argantonio y sus elementos míticos". Archivo Español de Arqueología, 87: 7-20.

Pérez González, J. (2017): “Aurifices en la Roma Julio Claudia. La fiebre del oro romana”. Studia Antiqua et Archaeologica 23(1): $37-70$.

Pérez González, J. (2019): "How Roman Sumptuary Specialists Named Themselves: A Corpus-Based Study”. Latomus, 78.

Pérez González, J. (2020): “Depredadores romanos. En busca del oro”, en: V. Revilla Calvo, A. Aguilera Martín, Ll.Pons Pujol, M.García Sánchez (eds.), Ex Baetica Romam. Estudios sobre economía, sociedad e instituciones de la Antigüedad. Homenaje al profesor José Remesal Rodríguez. Barcelona: Publicacions de la Universitat de Barcelona.

Pérez González, J. (en prensa): "The singularity of Rome. The Sumptuary city”. En: ICCA-AIAC 2918 19th International Conference of Classical Archaeology: Archaeology \& Economy in the Ancient World. Colonia-Bonn.

Quiring, H. (1948): Geschichte des Goldes. Die Goldenen Zeitalter in inher kulturellen und wirtschaftlichen Bedeutung. Stuttgart: Ferdinand Enke Verlag Stuttgart.

Rodríguez-Almeida, E. (1986): "Alcune Notule Topografiche Sul Quirinale Di Epoca Domizianea“. Bullettino Della Commissione Archeologica Comunale Di Roma 91, no. 1:49-60.

Rodríguez Almedia, E. (1994): "Producción y logística de algunos bienes: el caso de Roma”, en: X. Dupré Raventós (ed.), La ciutat en el món romà: XIV Congrés Internacional d'A rqueologia 
Clàssica: actes $=$ La ciudad en el mundo romano: XIV Congreso Internacional de Arqueología Clásica: Tarragona, 5-11/9/1993: Actas. Vol. 1. Tarragona: Institut d'Estudis Catalans: 335-346. Rostovtzeff, M. (1937): Historia Social y Económica del Imperio Romano. Madrid.

Rosman, K.J.R., Chisholm, W., Hong, S., Candelone, J.-P., Boutron, Cl.-F. (1997): "Lead from Carthaginian and Roman Spanish Mines Isotopically Identified in Greenland Ice Dated from 600 B.C. to 300 A.D”. Environmental Science \& Technology, 31(12): 3413-3416.

Sastre, I., Orejas, A., Currás, B., Zubiaurre, E. (2017): "La formación de la sociedad provincial en el Noroeste hispano y su evolución: civitates y mundo rural". Gerión 35(2): 537-552.

Terpstra, T.T. (2019): "Roman technological progress in comparative context: The Roman Empire, Medieval Europe and Imperial China". Explorations in Economic History. 